\title{
Prostate Cancer Care and Survivorship (CaPCaS) Model for Black Men: An Ethnographical Study of Prostate Cancer Survivors
}

FOLAKEMI T ODEDINA ( $\square$ fodedina@cop.ufl.edu )

University of Florida https://orcid.org/0000-0003-3796-1385

Mary Ellen Young

University of Florida

Deidra Pereira

University of Florida

Dagne Getachew

University of South Florida Tampa Official Bookstore

Christopher Williams

Urology Consultants Inc

Kenneth Stokes

Florida CHW

Parisa Fathi

University of Florida

Nissa Askins

University of Florida

Jennifer Nguyen

Mercer University College of Pharmacy

Ernest Kaninjing

Georgia College

Research article

Keywords: CaPCaS, prostate cancer, survivorship

Posted Date: August 26th, 2020

DOI: https://doi.org/10.21203/rs.3.rs-60444/v1

License: (c) (1) This work is licensed under a Creative Commons Attribution 4.0 International License.

Read Full License 


\section{Abstract}

Background: In 2020, 191,930 men will be diagnosed with prostate cancer. The lives of these men will change dramatically as they go through the prostate cancer care and survivorship process. Black men are especially affected, as they are more likely to get and die from prostate cancer. The primary objective of this study was to explain the experiences of Black men across by developing a prostate cancer care and survivorship (CaPCaS) model.

Methods: Based on the principles of community engagement research and employing qualitative methodology, we interviewed Black prostate cancer survivors to document their CaPCaS experiences relative to prostate cancer prevention, detection, diagnosis, treatment, survivorship and advocacy using audio and video recordings. Our data analysis plan included preparing and verifying the narrative data, coding data, and developing an interpretive framework for Black men's experiences across the prostate cancer care continuum.

Results: Thirty-two prostate cancer survivors participated in the study. A CaPCaS model was created with themes specific to the trajectory of prostate cancer prevention, screening, diagnosis, treatment, survivorship, and advocacy. Contextual themes identified were African Diaspora, Masculinity and Sociodemographic factors. Additionally, we identified cross-cutting factors across the CaPCaS process that included Acculturation, Self-efficacy, Health literacy, Patient-provider racial concordance, Stigma and Spirituality.

Conclusion: The CaPCaS model is an explanatory model of prostate cancer care and survivorship factors for Black men and will foster better understanding of behaviors associated with improved prostate cancer outcomes in Black men.

\section{Introduction}

Prostate cancer ( $\mathrm{CaP})$ is the sixth leading cause of cancer deaths globally and second relative to cancer incidence rates among men [1]. In the United States (US), the American Cancer Society estimates that 191,930 men will be diagnosed with CaP and 33,330 men will die of CaP in 2020 [2]. The State of Florida in the US is one of the leading states with respect to CaP burden. As of 2020, Florida ranks $2^{\text {nd }}$ in estimated new CaP cases with 13,950 new cases and $2^{\text {nd }}$ in estimated $\mathrm{CaP}$ deaths with 3,570 CaP deaths among the 50 states in the US [2]. Disparities in cancer also continue to be a major problem in Florida, especially for Black men. For example, Black men were the only racial/ethnic group that did not meet the US Healthy People 2010 objective to reduce the CaP death rate to 28.2/100,000 men and the only racial/ethnic group that has not met the Healthy People 2020 objective for CaP to reduce the CaP death rate to $21.2 / 100,000$ men [3]. The Healthy People objective for CaP is seen as a moving target for Black men, which may not be achieved due to multiple factors, including individual personal factors. Unfortunately, the CaP experiences of Black men in Florida is a microcosm of the CaP experiences of Black men globally. 
The CaP care and survivorship (CaPCaS) process describes the experiences men go through from $\mathrm{CaP}$ prevention through screening, diagnosis, treatment and survivorship. Currently, there is limited published research on the CaPCaS experiences of Black men, especially the psychosocial effects and coping mechanisms used by Black men. Some of the CaPCaS experiences that have been highlighted in the literature include shock when diagnosed with CaP [4], perception of "death sentence" [5], receiving helpful information from physicians that helped with anxiety [6], receiving inadequate information to make informed decision about treatment [7] and receiving limited treatment options that is usually tailored to the physician's expertise. Considering that patients going through the CaPCaS process will be experiencing different emotions and may not be prepared or have the knowledge to be fully involved in their treatment decisions, it is not surprising that some patients have decisional regret [4].

Decisional regret has been found to be associated with adverse events experienced by patients. When asked to discuss the impact of CaP treatment on their lives, most men reported several physical disruptions including urine flow issues and Erectile Dysfunction $[8,9]$. In one study, men shared the experience of having to receive a catheter [10] and how this affected their "sense of self" and disruption to sexual functioning. Another man stated the anxiety he endured as he dealt with incontinence and the use of pads [10]. While some men share intimate details of how the side effects of treatment impact their lives, other studies report that when discussing post-treatment, men tend not to dwell on the impact and put more emphasis on proceeding with life and gaining previous physical control of their lives $[8,11]$. The transition to CaP survivorship can be mentally and physically trying, especially for those who lack emotional and financial support. The spouse/partner can play a very important role in the decisionmaking process following diagnosis. Most men indicated that a spouse/partner was a significant support resource and they valued their opinions in the treatment selection process [6,12]. Family involvement and interaction with men experiencing $\mathrm{CaP}$ treatment has also been found to be important $[5,7,11]$. Maliski et al reported the role of faith/religion in coping with the initial reactions of shock in diagnosis and perceptions of fear [5]. Faith was concluded to be a source of empowerment, encouraging the men to be active in their treatment and to use their overall outcomes as meaningful events in their lives.

A significant gap in the literature on $\mathrm{CaP}$ disparities is documentation of the CaPCaS experiences of Black CaP survivors. Specifically, no study has employed Grounded Theory methodology to study the CaPCaS process among Black men. Furthermore, most studies on Black men's CaP care experiences have focused only on CaP diagnosis, treatment and survivorship, disregarding the important contributions in the areas of CaP prevention, detection and advocacy. The result of the limited research on CaPCaS process is the lack of tailored behavioral intervention programs focused on assisting Black men through the CaPCaS process. The primary objective of this study was to explain the experiences of Black men across the $\mathrm{CaP}$ care continuum through a CaP care and survivorship (CaPCaS) model. Based on the principles of community engagement research (CEnR) and employing qualitative methodology, the following study aims were achieved: (1) Recruited and interviewed Black CaP survivors, including nativeborn Black men (NBBM), African-born Black men (ABBM) and Caribbean-born Black men (CBBM); (2) Documented the CaPCaS experiences of the Black CaP survivors relative to CaP prevention, detection, 
diagnosis, treatment, survivorship and advocacy using audio and video recordings; and (3) Developed a CaPCaS model to explain the experiences of the Black CaP survivors.

\section{Methodology}

The primary objective of this study was to document the CaPCaS experiences of Black CaP survivors regarding CaP prevention, detection, diagnosis, treatment, survivorship and advocacy. The study setting was Florida (US). The study populations were NBBM, ABBM and CBBM with personal history of CaP. The research design selected for this study was prospective qualitative design because little is known about the CaPCaS experiences of Black men. Grounded Theory was the preferred qualitative method as it facilitates the development of theories about CaPCaS from participants' narratives. Participants were identified through the through the Florida Cancer Data System (FCDS) database.

\section{Participant Recruitment}

Following appropriate Human Subjects Review and approvals from the University of Florida, Florida Department of Health and the USAMRMC Office of Research Protections (ORP) Human Research Protection Office (HRPO), the study team worked with the FCDS to obtain a list of 1,813 patients diagnosed with $\mathrm{CaP}$ in 2010 . Following the recruitment strategies outlined by the Florida Department of Health (DOH), participants were recruited as follows:

1. Initial patient contact by written correspondence, including a letter from the $\mathrm{DOH}$, patient contact letter to request the patient's active consent for participation and patient response form.

2. For those who did not respond after three weeks from sending the initial (first) mail, a second mailing was sent with the addition of a telephone opt-out card.

3. For those who did not respond to the second mailing and the telephone opt-out card after three weeks, a study staff contacted them by telephone to introduce the study.

Participants who agreed to participate in the study were pre-screened to select eligible participants from the database provided by FCDS. The inclusion criteria for the project were: (1) NBBM, ABBM, and CBBM; (2) personal history of $\mathrm{CaP}$; (3) ability to complete two separate interviews with each one expected to last 2-3 hours; and (4) flexibility to meet interviewers at a convenient community site for the interviews. The pre-screening items included: name and contact information; country of birth; age; marital status; and education level. The demographic information was needed to facilitate a purposeful systematic selection of Black men of diverse age groups (less than 50 years versus over 50 years), marital status (single, including divorced or separated, versus married/in a relationship) and educational levels (college degree versus not college educated). The pre-screening phone survey was conducted by two Community Health Workers (CHWs) with additional support by staff of the University of Florida (UF) Survey Research Center (UFSRC) due to the volume of the pre-screening phone calls. An incentive of $\$ 5.00 \mathrm{gift}$ card was provided to all potential pool of participants. Once participants were selected, they were contacted to confirm: (i) 
their continued interest to participate in the study, (ii) ability to complete two (initial and follow-up) separate interviews, (iii) agreement to audio and video record the interviews; and (iv) ability to meet interviewers at a convenient community site for the interviews. Subsequently, interview times were set up with the participants.

\section{CaPCaS Interview Guide}

The study's in-depth interviews focused on the following phases of the cancer survivorship continuum:

- Prevention - Participants' understanding and practices of healthy behaviors prior to and after diagnosis, level of personal/family/physician involvement in prevention;

- Detection - Participants' understanding of risks and benefits of screening prior to diagnosis, screening practices prior to diagnosis, current thoughts about screening, current screening practices, level of personal/family/physician involvement in detection;

- Diagnosis - Participants' personal story of diagnosis, feelings, emotions, reactions, and/or regrets; understanding of the disease, prognosis and treatment options; level of personal/family/physician involvement in diagnosis;

- Treatment - The level of personal/family/physician involvement in treatment decision, process of treatment decision, types of treatment including non-medical treatments, understanding of treatment and side effects, decisional regrets (if any) about treatment;

- Survivorship - In this study, survivorship is defined as post-treatment survivorship. It was operationalized as the understanding of and comfort with uncertainty with $\mathrm{CaP}$, understanding of prognosis, level of personal/family/physician involvement in survivorship, current support/services available for care, challenges/successes related to survivor care, trust in clinicians, experiences with health care and follow-up treatment, experiences with late effects of treatment and second cancers (if applicable), description of quality of life, dealing with recurrence (if applicable), challenges/successes dealing with aggressive disease (if applicable), and challenges/successes dealing with advanced $\mathrm{CaP}$ (if applicable).

- Advocacy-Understanding of why some survivors choose to become advocates, characteristics of advocates, the process of becoming advocates, experiences as advocates, and impact of their advocacy role on their survivorship.

The study team worked closely with the study Community Advisory Board (CAB) members, comprising ethnically-diverse CaP survivors and advocates, to generate the items for the interview guide questions. The CAB members were provided the above definitions and then asked to think of relevant questions that can be asked to elicit information from Black men. The team met over several weeks by phone, discussing each factor and generating appropriate items. Once all the factors had been discussed, the study Principal Investigators reviewed all the questions and revised appropriately. The interview guide was then forwarded to the CAB members again for final review. To prepare for data collection, mock 
interviews and pilot interviews were conducted by the $\mathrm{CHW}$ interviewers using the interview guide (available as supplemental research material).

\section{Data Collection}

The CaPCaS interview guide served as the data collection tool for the project. We planned for a total time of about 5-6 hours for data collection per participant, sufficient for gathering in-depth perspectives on the research questions. In order not to create burden for our participants, we scheduled two interviews lasting not more than 4 hours at a time. Participants had the choice to have the interviews spread over two days. Prior to the first interview session, one of the CHWs conducted the informed consent process, obtained the appropriate signatures, and provided the participant a copy of the consent form. The interview commenced immediately following the informed consent process. Using the identified topics as a guide and also taking cues from each interview, the in-depth questioning of participants was conducted by the assigned $\mathrm{CHW}$. Although each interview was personalized to each participant, all the topics in the CaPCaS interview guide were covered.

Data was collected over two separate interviews to ensure that all interview topics were covered. All interviews conducted on Day 1 and Day 2 were audio recorded to provide ease of transcription and back up of data. During the first interview, consent was obtained from participants who were good candidates for the video portion of the study. For the participants who consented to additional video interview, audio and video were used to record the second interviews. Participants were compensated appropriately for participating in this study. All participants received $\$ 75$ for the first interview and $\$ 75$ for the second interview (total of \$150). In addition, participants who participated in the video interviews received an additional $\$ 25$ after the second interview.

\section{DataManagement and Analyses}

Our data analysis plan included preparing and verifying the narrative data, coding data, and developing an interpretive framework for Black men's experiences across the CaP care continuum. Using an iterative process, the team developed the interpretive framework to develop the explanatory CaPCaS model.

Interview transcriptions: Interviews were transcribed verbatim by a professional transcription service that has policies in place for the protection of Protected Health Information (PHI). Each transcript was then verified for accuracy by a study team member.

Data coding, modeling and analyses: The qualitative methodologist and research assistant were responsible for downloading the audio and video files, sending the audio files to the transcription service, downloading the transcripts when returned, making sure the verification was completed, and importing the transcript and field note files into the NVivo 10 software, a computer-assisted data analysis software (CADAS) that allows coding and modeling of complex narrative data. While the NVivo 10 software does 
not analyze the data, it provided a sophisticated and systematic way to manage the tasks of: (1) organizing large quantities of narrative data, (2) coding text, (3) retrieving text by codes, (4) querying the data, (5) comparing sets of data interpretation across samples and (6) developing analytic models. Led by the lead qualitative methodologist, the data coding was conducted by our study interdisciplinary team. Team members were asked to write and share reflexivity statements, so that perspectives of all team members were understood. The study team members coded the data in weekly team meetings. The coding consisted of reading the data and identifying major themes, then assigning labels to and defining emerging categories. The qualitative methodologist and research assistant then coded the data in NVivo 10 to reflect team coding decisions.

Two levels of coding were conducted. The first, open coding, refers to an approach to data with no preconceived ideas about what will be found and the second, focused or axial coding, refers to reviewing data for the purpose of more richly coding on a particular theme [13]. Dimensional analysis was employed to make sure that each emerging concept was carefully defined. The study team went back and forth between the data and the emerging analytic framework, employing constant comparison of new data with already coded data and new categories with previously analyzed text [14]. To ensure trustworthiness and credibility [15], the study team maintained an audit trail that documented how and when analytic decisions were made. In addition, peer debriefing was conducted to ensure credibility, including the presentation of findings to the study CAB members as part of the CEnR approach.

\section{Study Results}

\section{Study Participants}

The FCDS provided a database of 1,813 participants identified as Black men diagnosed with CaP in 2010. The FCDS does not extract data on patients with a death certificate, thus we found out that a few of the men were deceased during the pre-screening phase. In addition, there were bad addresses. A total of 212 completed responses were obtained by phone. Figures 1-3 provide a pictorial summary of the demographic information of participants. A majority of the participants were between 60 and 69 years (48.2\%), had only high school diploma (26.1\%), and married (65.3\%). Relative to ethnicity, $67 \%$ of participants classified themselves as African-American of American origin (born and grew up in America), $20 \%$ as African-American of Caribbean origin (born in one of the Caribbean Islands but now American), $4 \%$ as Caribbean, 3.5\% as African-American of African origin (born in Africa but now American), $0 \%$ as African, and 5.5\% as Other/Don't know/Refused. For the CBBM, the most common countries of birth are Jamaica, Haiti and Guyana, respectively.

\section{Qualitative Findings}

Constructivist grounded theory methods [16] were used to explore the experience of CaP prevention, screening, diagnosis, treatment, survivorship and advocacy from the perspective of Black men in depth, 
giving participants the opportunity to provide a rich narrative and to explore both the positive and negative aspects of their experience. Forty-one participants (20 NBBM, 20 CBBM and 1 ABBM) were selected from the 212 participants to participate in the study. We had only one ABBM who agreed to participate in the interview phase. Selection was conducted systematically to ensure representation in terms of age, marital status, education and geographical location. Data saturation was achieved with 17 NBBM, 14 CBBM and 1 ABBM, after which we ended data collection. Data saturation is the standard for deciding that we are not finding anything different from the interviews first coded and last coded.

Due to the fact that only one ABBM was recruited, we are reporting demographical data for only the NBBM and CBBM. However, the interview data of the ABBM is included in this report. Table 1 provides the demographic information of participants. Majority of the participants are NBBM, between 50 and 69 years, do not have a college degree and are married. Over $80 \%$ of the participants did not have any support system with them at the point of CaP diagnosis. Table 2 provides examples of the themes identified for each of the CaP care continuum. 
Table 1

Participants' Demographic Characteristics**

Variable

Frequency $(\mathrm{N}) \quad$ Percentage

Ethnicity

Native Born Black men

17

54.84

Caribbean Born Black men

14

45.16

Age

Less than 50 years

1

3.23

50 to 69 years

23

74.19

70 years and above

7

22.58

Education

Less than college degree

20

64.52

College or Post-college degree

11

35.48

Marital Status

Now married

19

61.29

Widowed

4

12.90

Divorced

4

12.90

Separated

1

3.23

Never married

3

9.68

Presence of Family/Friends at Point of Prostate Cancer Diagnosis

None

Wife Only
25

5

16.13 
**Note. There was total of 32 participants with one (1) African-born Black man, who was not included in this table. 
Table 2

Themes/Intervention Messages Emerging from Participants' Interviews

\section{Prostate Cancer Care Continuum \\ Themes}

Prevention

- Need for advocacy message for prevention

- Need for and challenges of preventive lifestyle

- Limited awareness about prostate cancer

- Need for public awareness about prostate cancer prevention

- Need for education about prostate cancer prevention

- Lack of Knowledge and checkups.

- Perception of prostate cancer

- Importance of Black men's health

Screening

- Need for screening without symptoms (due to family history of other cancers)

- Impact of masculinity on screening

- Importance of testing

- Locus of control

- Screening importance for Blacks

- Overcoming Digital Rectal Exam (DRE) myth

- PSA controversy

- Importance of screening on survival

- Physician as cue for action

- Procrastination by Black men.

- Cue by wife

- Importance of prostate cancer education

- Take Command of my Own Health

Diagnosis

- Denial

- Cancer Fatalism

- Disbelief

- Thoughts about Impact on Manhood

- Questioning (why me?)

- Exploring reasons

- Emotional

- Shock

- Hope

- Fear

- Fight / Resilience

- Need for immediate action / Urgency

- Acceptance

- Religiosity / Faith

- Will to live

- Delay taking action

- Need to be comfortable with physician 
- Need for a second opinion

- Need for emotional support time to reflect on diagnosis

Treatment

- Treatment choices

- Locus of control

- Decision making - self reliance

- Decisional regret

- Decisional regret due to unrealistic expectation provided by physician

- Importance of social support, family support, asking for help, asking for support

- Making it your decision

- Shared decision making with physician

- Several consultations before treatment choice

- Information access

- Impact of adverse effects

- Acceptance of side effect

- Spirituality and medical knowledge working together

- Dealing with the gravity of diagnosis

- Informed decision making

- Unreliable sources of information

- Addressing side effects - unsatisfactory

- Learning from others' experience

- Limited discussion by Physician

- Perceived control I Taking control

- Importance of personalized care

- Important of knowledge in treatment decision

- Right physician

- Interdisciplinary team

- Treatment expectation | Positive attitude

- Partnership with provider

Survivorship

- Changing view of meaning of prostate cancer (from fatalism to survivorship)

- Lifestyle change

- Survivorship meaning and impact

- Support

- Face of prostate cancer

- Prostate cancer at early age - implication for recovery and recurrence

- Dealing with Adverse effects: Impotency

- Focus on surviving

- Continued monitoring

- Need for information for survivorship

- Connection with those "who look like me"

- Support for self

- Support for spouse

- Access to physician 
- Meaning of prostate cancer after diagnosis

- Dealing with depression

- Masculinity

- Self-efficacy

- Prevention

Advocacy

- Willing to speak to anyone

- Sharing prostate cancer journey

- Education advocacy

- Religiosity

- Information need

- Effects on family

- Faith

- Need for advocacy

- "Calling" to be an advocate

- Talking to family/sons

- Sharing experience with younger men

- Embracing advocacy

- Social support

\section{Study Interpretative Frameworks}

The Good, The Bad and The Ugly (Figure 4). The qualitative methodologist led the team in developing emerging frameworks from the data. The experiences of the Black CaP survivors across the CaP care continuum were divided by the study scientific team and the study CAB into "the good" (e.g. timely diagnosis, excellent provider relationships, informed decision making, effective treatment, no decisional regret) and "the bad" (delayed diagnosis, poor provider relationships, inappropriate decision making, less effective treatment, decisional regrets). Results were presented at several community forums for validation where participants were presented with preliminary findings and asked to provide feedback to the team. At one of these events, a Black CaP survivor stated that while we know a lot about the "good" and the "bad" experiences, we did not know about the "ugly." He defined "the ugly" as the fact that we know little about Black men who did not enroll in the study - men who may be the most vulnerable to health disparities due to lack of access to screening, physical debility, or death due to CaP. This construct was added to the overall experience model.

A Prostate Cancer Care and Survivorship (CaPCaS) mode/ was created representing the trajectory of CaP prevention, screening, diagnosis, treatment, survivorship, and advocacy (See Figure 5). The contextual factors participants reflected on included the immigration patterns of Blacks, including the African diaspora, the construct of masculinity of Black men, and socio-demographic factors. The sociodemographic factors include age, marital status, educational status, employment and income. Each phase of the trajectory can be described in terms of the "the good and the bad," that is contributing to 
satisfactory or unsatisfactory outcomes. CaP prevention and advocacy anchor each end of the process model, as these processes are in play but do not necessarily impact the experience of the disease directly.

Some of the unique themes that emerged for $\mathrm{CaP}$ prevention were the perception of limited awareness about $\mathrm{CaP}$ in the Black community and the need for public awareness/education. Other themes were the need for risk-reduction behavior, preventive lifestyle, health beliefs and cues for action. Participant 1086 described the importance of lifestyle, "It's very important for us to eat better and to leave a lot of that sodas, and drink more water, okay? It's very important for you to do that." The importance of public awareness was mentioned by participant 355: "Until recently, I don't think there are aware of it to be honest with you, but, um, recently more, um, of our daughters -sons and daughters - are more aware of it and it's more publicized now...". Another participant, P001, stated that "Black men as a whole need to become, uh, more aware of things that affect them primarily other than, uh, other races, prostate cancer being one of them". Also found to be important was health beliefs:

"Well, the first thing that comes to one's mind when they hear the-the big letter $C$ is that, "Oh, man. This is a journey. It's a journey that can either put you in the grave or it's a journey that you're just gonna have to fight vigorously, but your life is basically changed." And so I had to look at it-l'm a-as-as-if-if it's was gonna kill me, I gotta go down fightin' with it. But it does change your life". [Participant 866]

"I think we-we really have a negative view of it, and then, too, even though it's negative, it's not something that's really taken seriously. Uh, I think, uh, cancer is a serious thing, but I don't think we take prostate cancer serious. When-when-when-when the doctor says you have a elevated PSA count or somethin' like, and-and it's, like, 'Okay. So what?' [Participant 1881]

The factors that emerged for $\underline{\mathrm{CaP}}$ advocacy. included helping others, sharing experiences and embracing advocacy. A few of the men considered themselves as CaP advocates and expressed their willingness to share their personal CaP journey with other Black men. Some discussed their CaP diagnosis as a "calling to become a CaP advocate" and thus have embraced advocacy, especially by sharing information about the disease. Some of the men also brought up providing social support for newly diagnosed Black men:

"I'm not ashamed of it. I'm ready to discuss it anytime, anywhere because if that way helped them -- for that is another reason why I do this - if that way helped them, that's very good. So they don't have to go through what I went through". [Participant 355]

"I would consider myself an advocate because from personal experience, you know? It's easier to talk to someone about something, but when your personal experience - it really opens up for you because I'm not just talking about stuff that I read or heard people. It's from personal experience, and that makes you, you know, makes it more legit". [Participant 1261]

CaP screening was discussed in terms of Digital Rectal Exam (DRE) and Prostate-Specific Antigen (PSA) screening tests. The sub-themes that emerged for DRE were 'unmanly', 'rape', 'overcoming DRE myth' and 
'just something you have to do' while the sub-themes that emerged for PSA were 'limited access', 'routine' and 'not recommended'. In general, participants emphasized the importance of screening in their CaP journey.

"Please go get checked. But, um, I-I-I feel that it's necessary, I mean, 'cuz, again, if you don't have those symptoms, then you won't know like me. I didn't have symptoms. My prostate wasn't enlarged. Um, okay, my prostate wasn't enlarged. Um, you know, I wa-I didn't have no, you know, bleedin' nowhere, none of that, none of the above, no-nothin'. So, you-gettin' screened is very, very important so you'll know." [Participant 1086]

"I'm a bit confused, because there's some recent literature that was made available to me that said that some scientists are now questioning the value of the PSA test and say really it's not what it's supposed to be. He got a false reading from it, so I don't know. I'm just confused." [Participant 2018]

"I was discussing with my mechanic, bending down for a doctor to stick their hand in your anus to feel it whether it's smooth or it's not rape... They just are examining you, your prostate, and if it is smooth then they don't need to do anything, fine." [Participant 355]

"In the beginning, I realized, you know, the screening portion, uh, it was scary. You know, all you hear is, when you hear the word prostate cancer you hear, uh, you didn't hear white guys dying. You were hearing the Black man disease, or the blacks dying at a higher rate because of stats saying they're not getting medical treatment, they're getting diagnosis late, or they're not adequate getting medical care. Those were the things I heard. Did I know anything about the disease? No. You just seen public service announcement: Black mens are dying from prostate cancer, you know, at a higher rate from their White counterparts." [Participant 2039]

"Um, maybe it-maybe it's-again, because of the fact that we're just very reluctant-in there's a lot of pride, so when you talk about goin' and somebody stickin' their-their finger in their rear end and so forth, it's-you know, those men are-they're so macho, they're not-they're-they're tryin' not to-to go there. Whereas, uh, in this country you're a little bit more educated and realize that this means life. And so I think that's what it is with the Caribbean islands, especially Jamaica. Um, those-those men down there are just a little bit too macho for their own good". [Participant 866]

The role of physicians and wives as cue for screening action was also highlighted by participants. Additional themes were the importance of annual physical to facilitate screening, procrastination that delays screening, symptom interpretation and temporal orientation. On symptom interpretation, participant 1086 stated - "Also pay attention to, um, you-your-your go-your bathroom use, you know, how often you wakin' up at night. Does it feel like you completin' your urine, um, stuff like that? And-and if that PSA level is going up, then go get checked". Temporal orientation $[17,18]$ is defined as an individual's perception of time as being in the past, present or future. Blacks have been noted to exhibit present temporal orientation. The implication in terms of health is that prevention and screening activities are limited when there are no symptoms. 
"I basically ignored it for almost 8 years. I basically just ignored it because my life was normal. Doctor told me that my prostate was enlarged, but that was not a problem. I went on television and saw they had prostate pills so I ordered some of those thinking yeah take the pills and everything's gonna be fine. And everything was fine! I was not sick. Didn't feel any way ill. So my life was normal, there was nothing abnormal about my life except when I went to the restroom to urinate sometime and it was slow, but that was it I thought it was no big deal. So your PSA count is high? What does that really mean? It meant nothing to me because it was not affecting me in any way". [Participant 1881]

"And the thing [the doctor] would tell me on the PSA, 'Well, you-you need to check with urologist. You need to go ahead and have this checked out.' And I would say, "Okay. I'll do it.' Never-never did it. Never did it. I mean this went on for, like, two or three years. Just never went. No big deal. I mean I was havin' trouble urinatin' at times, uh, but it was-I thought it was no big-you know, it was nothin' to get alarmed about". [Participant 1881]

For CaP diagnosis, the men's discussion revolved around treatment decision and outcomes. The need to be comfortable with the physician at the time of diagnosis, emotional support, and time to reflect on diagnosis were key themes. A crucial point for all the men within the CaPCaS process is the Point of Prostate Cancer Diagnosis (PPCD) $[19,20]$. As part of the CaPCaS process, we found that Black men come to the PPCD with different emotions and different experiences. The different PPCD emotions include shock, hope, fear, resilience, need for immediate action, acceptance, religiosity / faith and the will to live. Additional factors identified at the PPCD were denial, cancer fatalism, disbelief, and thoughts about impact of disease on manhood. Participants also expressed the need to be comfortable with the physician, the need for a second opinion, the need for emotional support and the time to reflect on their diagnosis at the PPCD. Faith and the will to live emerged as coping mechanism during CaP diagnosis phase. Some of the comments by the men were:

[Biopsy scare, Participant 1086] "I mean, biopsy is tough. I'm gonna tell you right now. It's-it's a tough muster. It's tough, but it was necessary".

[Spirituality, Participant 1086] "I mean, um, it-put it in the hands of God, really, you know, and-and-and, um, let him lead you".

[PPCD Support, Participant 2039] "And I found it is good to take someone with you who's been through it, even if they sit there and don't say nothing. That's somebody who, when the doctor tell you, "You got cancer"... When they told me, I didn't hear him. I didn't hear him! My wife was in there when the man said cancer. Everything after that sentence I didn't hear. So my point is, you take someone who's going to hear what they say so they can repeat it to you when you get away and get your mind back and say 'What did they say?' They can repeat it to you because you're gonna miss - I don't care who you are. Once that doctor says 'it came back positive and we gotta do this', your mind goes everywhere except listening to what that cat say. And then when you finally listen, your mind is so screwed up on 'am I gonna die? How bad is it? Are you gonna lose your friend?"'. 
[Sharing diagnosis, Participant 2039] "On Christmas Day, I said 'Mom, I got something to tell you'. All the family was here, so I said I got to tell the family. So I made an announcement that I was diagnosed with prostate cancer. They knew I had been sick, having some problems, but they did not know why. So I had we was all at my house at the table and I told my mom, I said, 'Mom, I got diagnosed with cancer, prostate cancer' and -you think the room go quiet, everybody go hush - but then my brother -- he's 2 years older than me -- he was here. He came to the table and said, 'Mom, I got something to tell you'. Now you got to understand this was a festive occasion, but this was when all the nieces, nephews, brothers, sisters, wives, everybody here. Now he came to the table, 'Mom, I got diagnosed too'. And when he dropped the bomb, it was more or less 'I didn't know you had it! I didn't know you had it!' And it goes back to this thing where we keep it all to ourselves, but he took the opportunity to share with the family. And the thing that made it all ironic - I said it earlier and I'll say it again - we both went through it. We both was able to compare notes, you know. I didn't really get into it because of him, but we talked, we passing in the wind. I'm at the treatment center in the morning, he got off of work, he in the evening."

For the CaP treatment phase, the importance of information processing, help seeking, shared-decision making with a physician, support, perceived control, provider communication, positive outcomes and learning from the experience of other CaP patients/survivors emerged from the interview data. In addition, provider bias and adverse effects were identified. Participant 1086 justified his choice of surgery as "I just wanted it outta me. I wanted the cancer outta me" and he was " 100 percent satisfied with my decision". Trusting the physician was clearly expressed:

"I ain't got no family here in with me, so I'pend on my-on my-my doctor, which I had ever since I had -I been going to this doctor ever since I've been for 15 years cuz, you know, you-you-you-you know, we close, and he always-you know, if somethin' come up, he always tell me, you know, "Let's go and check this out," or "Go-let's see what this is about" or-you know. And I go ahead and do it". [Participant 798]

"The advantage of having home boy or home girl as your doctor-...-is that you speak the same language. On weekend, you can go to their house and ask free questions which you don't pay for". [Participant 355]

\section{Another theme that came across clearly was the need for second opinion:}

"Um, I was disappointed with the, um, original urologist who I went to see, um, primarily because after he diagnosed the cancer and-and told me, um, you know, the severity of-of-of how much it had spread, uh, he gave me only one option, and that was radical surgery. Um, when I asked him about other forms of treatment, he sort of dismissed the question, uh, and led me to believe that the only treatment that was available to me would be the radical surgery. Um, I didn't necessarily agree with that, and so-[clears throat]-I did some additional research, talked to some other urologists, um, and decided to actually change urologists, uh, to a different urologist, who would provide either type of treatment that I decided that I wanted. I decided, um, to go with radiation therapy and seed implant, uh, mainly because I felt that 
it would be the safest, uh, procedure, um, and have the least, um, negative effects, uh, on my life". [Participant 449]

\section{The decision-making process and outcomes were clear drivers of CaP treatment:}

"You have to come to a decision ...it is a life or death decision". [Participant 1086]

"You are somewhat baffled -I mean, here is something that you have not experienced before. You do want to make the right decision. Uh, the way I was set up was I had, uh, doctors from different areas of treatment to, uh, talk with me. There was a surgeon, there was an oncologist, and there was, uh, one other person that, uh, did uh... The urologist, yeah. So all of them will, uh, talk with you -- and of course, they have your history in front of them - they will try to help you make the right decision. However, uh, Surgeon, of course, he's promoting surgery, and, uh, the oncologist gives you other options. The final analysis is kind of left up to you depending on, uh, your family history or your state of health". [Participant P001]

"You take anybody word that's in a white coat. Now, I don't take nobody word just from my experience -not to say that I know everything. I'm saying by my experience on misdiagnosis, different side effects it's important when somebody tell me "now you got this," I want to know how is this going to affect me down the road. Am I going to be better or am I going to be worse? If I'm going to be worse, then why am I doing this? When I don't understand - what I want people to understand is, when you don't understand something, don't leave the office like I did. I left the office in the first couple of years - this is my fourth year - the first two years, I just do whatever Joe Blow or Mary Ann say, I thought it was God. Now, in these last two years, I'm not taking it anymore. I'm' asking the questions because the quality of life is what you want to know. How is my quality of life going to be better with the decision they say you need?" [Participant 2039]

\section{Support and positive attitude were expressed as being helpful:}

"I knew I had to have a positive attitude about this because it had been explain to me that the more positive I was, uh, the more successful our treatments would probably be, so I had to have a very positive attitude with what was going on with me". [Participant P001]

"My family was involved with me through the entire process. I explained everything that was going on with me to them. And I was, uh, blessed enough to have a doctor-one of my daughters is, uh uh, has a doctor of pharmacy. She's a Pharm. D, and she was very helpful with me, uh, in explaining the process and telling me why they were doing this and why they did that and and the other. So, uh, family involvement was very important. I kept them involved, uh, in what was going on with me. However, I did not require them to transport me to and fro. Uh, I could do that on my own". [Participant P001]

If there is one primary thing that is clear in the CaPCaS process, it is that "there is no stupid question": 
"One of the things that I found out now is it ain't no stupid question. You ask and you ask and you ask till you get the answer. I found out they don't like it, but I make it clear to them I don't understand. Can you -I have a tendency to tell the doctor "Can you talk down to my level and say it in English?" And they'll laugh at it - you make a joke of because one we have a tendency - we don't want to upset the doctor, we don't want to offend the doctor, we don't want to get a second opinion. Here's the problem: it's your damn health. You can't worry about offending them. There's only two options: they say "I don't want to see you no more" or "I'm going to refer you to another doctor." At the end of the day, you must ask those questions. If you don't - I repeat it again - if you don't understand something they saying, you raise your hand. "Excuse me sir, I know you done said that." I say, "Help the old dog out. I don't understand. Can you bring it down a little?" They be like, "Mr. So-and-so, you crazy." I break it down to them. I understand - I want to know how this will affect me. When I talk to someone in the street, I tell them you don't want nobody to talk above you or act like they know everything. When I talk to my friends or church members, I tell them the same thing. When you go, I tell them what to ask, what I been through, don't be afraid to ask no question because it ain't no stupid question. The doctor may say, "I explained that two or three times." You getting offended? This is a decision that can have very bad, uh, very bad decision on you and your family if you don't make the right health decision". [Participant 2039]

Post-treatment $\mathrm{CaP}$ survivorship generated a lot of discussion among the $\mathrm{CaP}$ Black survivors. $\mathrm{CaP}$ survivorship was discussed in terms of side effects and decisional regrets. One of the key themes that emerged is the need for lifestyle change in terms of secondary prevention. The men also discussed challenges they experienced during survivorship, including dealing with treatment side effects (such as impotency), continuous monitoring (especially for recurrence) and dealing with depression. Decisional regret was linked with unrealistic expectations provided by the physician.

"My physician told me, "Okay, 6 months to a year, you'll be back to normal." That's what he told me. "6 months to a year, you'll be back to normal." He kept preaching that, he didn't tell me anything different. He told me [emphasizes] 6 months to a year you'll be back to normal." So when I, when I went into surgery, I went into surgery with that in mind - "Hey doc, in 6 months to a year, you'll be back to normal, okay, and you won't have that problem." [It's] still ongoing. It's almost 5 years, and it's not back to normal".

[Participant 1881]

\section{As expected, the adverse outcomes arising from treatments presented some challenges for the men during survivorship:}

"Well, number one, you've always been in control of your toolbox. You know? So you're gonna have to learn how to walk all over again. And there's a 90 percent chance, I will tell you-they say 50/50. I will tell you a 90 percent time that it might not come back, and so you're gonna have to use-there's times when, you know, it would come back on its own. When we gonna have sex, it would go up on its own, but it doesn't sustain. Most of the time it would go up on its own, but it might not sustain. But one of the things that have helped us is the pump". [Participant 866] 
"Well, one of the side effects is that, uh, that the radiation, the constant radiation in that area tends to weaken certain muscles in that area, okay. So you have to be careful. When you-you have to make sure that when you-when you leave home that everything that you needed to have done before you left home, do it. You know". [Participant 329]

"The incontinence-yes, it does weigh on you because, again, we're men, and we don't wanna go around knowin' that we have to be wearin' Pamper and all of that. Now get this. When I came out, they-the doctor said that I had to be out of work for six weeks. I had the bag on my leg and so forth or whatever, and after two weeks I went back to work with the Pamper. And the reason why is because, again, I ran a business and so forth, and I-I had to be there just to make sure that I had a business when I came back". [Participant 866]

The need for helpful information on CaP survivorship as well as ability to connect with other Black men who have undergone the same experience were noted to be helpful in the survivorship phase.

Also crucial to understanding the CaPCaS process are the crosscutting factors identified by participants and listed at the bottom of the model. These factors emerged as themes across the CaP care continuum and include family history of cancer, information seeking, acculturation, locus of control, self-efficacy, conflicting interests, health literacy, knowledge, comprehension, access to care, patient-provider racial concordance, patient-provider relationship, spirituality, stigma, positivity and social support. It appeared that provider communication style (paternalistic versus shared decision-making) impacted participants' relationship with their providers. The phenomenon of locus of control emerged multiple times as participants struggled with control while going through the process.

"After I was diagnosed and the treatment decision, it was in the beginning, it was left up to the doctors. You basically take they word on what you should do with your body. I didn't have much input. I had guys that say you "need to do this, you need to go over here and do that" and what I'm saying is, today, now that I took control of what I think is best for me as far as treatment, it's important to me -- I don't know about everyone else, but it's important to me -- I am making sure when someone say "we going to do this," I'm asking the question, "Okay, if you do this, what is the success rate? If you do this, how is this going to give me a quality of life? Is this going to better off doing this procedure or am I going to be worse?" It's very important in my life now to be able to ask those questions. I didn't ask in the beginning. If I asked those questions in the beginning, I promise you I wouldn't be going through half of the stuff I'm going through now. It was a lack of knowledge, not being able to have input or info". [Participant 2039]

\section{Also of primary concern is access to care, especially in terms of health insurance:}

"And I think-but I was guided-I think I was led that way by-by options that were not viable, that were not given to me with the-once again, it was the policy beginning to lapse, not bein' renewed, not knowin' whether or not I'm gonna be able to get insurance to-to follow through on that. And so there's an urgency 
situation here as well. "I gotta get up under this before that policy lapse and get this done." [Participant 1881]

\section{Discussion}

Our findings confirm some of the factors previously noted in the literature. Similar to our findings, lack of $\mathrm{CaP}$ knowledge, beliefs about CaP, and cultural norms associated with mistrust of the healthcare system were linked to screening [21,22]. A mixed method longitudinal study of 277 Black men found that nearly half $(44.8 \%)$ of the participants were not aware of the PSA, and approximately $27 \%$ had not heard of the DRE [23]. Lack of knowledge was often attributed to a lack of culturally appropriate health messages [21,23-25]. Additionally, greater levels of knowledge were associated with lower decision conflict and greater likelihood of talking with their physician about CaP testing $[25,26]$. Another qualitative study which conducted individual focus groups with men and their significant others found that female significant others were seen as key motivators for men to be screened [28]. Also similar to our findings, CaP testing and treatment have been reported as a threat to men's sense of masculinity [29]. Informed Decision Making (IDM) or Shared Decision Making was a recurrent theme in our study and has also been documented in the literature. Interventions that explored IDM, found that men were more likely to talk to their physician about $\mathrm{CaP}$ after receiving some form of health education [27]. Church led interventions were identified as having great potential to increase informed decision making among men [30]. Similar to the impact on screening, the presence or absence of a spouse at consultations also had implications for men's engagement with healthcare systems [31].

Overall, the CaPCaS model summarizes factors of significant importance to Black men across the CaP care continuum. For the numerous Black men who receive a diagnosis of $\mathrm{CaP}$, their lives change dramatically as they go through the CaPCaS process. Although, each man's CaP journey is unique to him and never the same with any other man, the ability to connect with others and learn from others who have gone through the same diagnosis is often referred to as "medicines that doctors cannot inject and cancer

cannot defeat'. The themes identified for each of the CaP care continuum factor (prevention, screening, diagnosis, treatment, survivorship and advocacy) as well as the crosscutting themes provide critical information that can be targeted to support Black men. For example, we found that the point of diagnosis is a crisis point for Black men and a critical point for intervention. The PPCD experiences of Black men provide information that can be used by physicians to prepare for their PPCD consultation with Black men as well as develop a support system for Black men.

\section{Conclusion}

The Florida CaPCaS project represents a major investment to address the health disparities and increased burden of morbidity and mortality of Black men diagnosed with CaP. The primary limitation of the study is that we were able to recruit only one ABBM for the study. This is because the cancer registry data, which was the primary source for participants lack data on the country of birth of patients. As has been consistently documented by our research group, there is significant within-group differences among 
Black men [21,32-34], which has unique implications for addressing the problem of CaP disparities in Black men. Future studies need to increase the representation of Black immigrants/foreign-born Black men in CaP disparity projects.

The CaPCaS model is an explanatory model of CaP care and survivorship factors among Black men and will need to be validated in future studies. Once validated, it will foster better understanding of behaviors associated with better CaP outcomes in Black men. In addition, it will provide evidence for the design of culturally responsive, tailored, targeted, and sustainable intervention programs to educate and improve the CaPCaS process.

\section{Declarations}

\section{Ethics Approval and Consent to Participate}

Human Subjects Review and approval were obtained from the University of Florida (Study ID IRB201300210), Florida Department of Health (DOH IRB Number: H13093) and the USAMRMC Office of Research Protections (ORP) Human Research Protection Office (HRPO \# A-17814)

\section{Consent for Publication}

Not Applicable. The manuscript does not contain any individual person's identifiable data in any form.

\section{Availability of Data and Materials}

The datasets analyzed during the current study are available from the Prostate Cancer Transatlantic Consortium (CaPTC) through the corresponding author. Data requests for collaboration with CaPTC on this dataset should be sent to captc@cop.ufl.edu.

\section{Competing interests}

No financial or non-financial competing interests.

\section{Funding}

The program was funded by the Department of Defense Prostate Cancer Research Program of the office of the Congressionally Directed Medical Research Programs (Award W81XWH-13-1-0473).

\section{Authors' Contributions}


CRediT roles: Conceptualization; Data curation; Formal analysis; Funding acquisition; Investigation; Methodology; Project administration; Resources; Software; Supervision; Validation; Visualization; Roles/Writing - original draft; Writing - review \& editing.

- FTO: Conceptualization; Data curation; Funding acquisition; Investigation; Methodology; Project Supervision; Validation; Visualization; Writing - original draft

- MEY: Conceptualization; Data curation; Formal analysis; Investigation; Methodology; Project administration; Supervision; Validation; Visualization; Writing - review \& editing.

- DP: Formal analysis; Investigation; Methodology; Validation; Writing - review \& editing.

- GD: Investigation; Validation; Visualization; Writing - review \& editing.

- CW: Investigation; Validation; Visualization; Writing - review \& editing.

- KS: Project administration; Visualization; manuscript review.

- PF: Visualization; Writing - review \& editing.

- NA: Visualization; Writing - review \& editing

- JN: Project administration; Visualization; manuscript review.

- EK: Project administration; Visualization; manuscript review.

\section{Acknowledgements}

The authors wish to thank Ms. Eva Egensteiner for her role in supporting the CaPCaS project. In addition, we would like to acknowledge the valuable contributions of the study community advisory board: Dr. Angela Adams, Mr. Oladapo Odedina, Mr. James West (late), Mr. Samuel Gaddy, Rev. Afolabi Ehikioya, Mr. Larry Pierre, Mr. Charles Griggs, Pastor Zacch Olorunnipa and Mr. Joey Johnson. Finally, we value the priceless contributions of all the study participants who allowed us to walk with them in their prostate cancer journey.

\section{References}

1. Cancer Today. In: Global Cancer Observatory. International Agency for Research on Cancer. 2018. https://gco.iarc.fr/today. Accessed 21 April 2020.

2. American Cancer Society: Cancer Facts \& Figures 2020. https://www.cancer.org/content/dam/cancer-org/research/cancer-facts-and-statistics/annualcancer-facts-and-figures/2020/cancer-facts-and-figures-2020.pdf (2020). Accessed 21 April 2020.

3. Florida Statewide Population-Based Cancer Registry. Florida Cancer Database System, Miami. 2020. https://fcds.med.miami.edu/inc/welcome.shtml\#. Accessed 21 April 2020.

4. Sinfield P, Baker R, Camosso-Stefinovic J, Colman AM, et al. Men's and carers' experiences of care for prostate cancer: a narrative literature review. Health Expectations. 2009; doi: 1111/j.13697625.2009.00546.x. 
5. Maliski SL, Connor SE, Williams L, \& Litwin MS. Faith among low-income, African American/black men treated for prostate cancer. Cancer Nurs. 2010; doi: 1097/NCC.0b013e3181e1f7ff.

6. Jones RA, Wenzel J, Hinton I, Cary M, et al. Exploring cancer support needs for older AfricanAmerican men with prostate cancer. Support Care Cancer. 2010; doi: 10.1007/s00520-010-0967-x.

7. Sinfield P, Baker R, Agarwal S, \& Tarrant C. Patient-centred care: What are the experiences of prostate cancer patients and their partners?. Patient Educ Couns. 2008; doi: 10.1016/j.pec.2008.05.001.

8. Gray RE, Fergus KD, \& Fitch MI. Two Black men with prostate cancer: a narrative approach. Br J Health Psychol. 2005; doi: 10.1348/135910704X14429.

9. Rivers BM, August EM, Gwede CK, Hart Jr. A, et al. Psychosocial issues related to sexual functioning among African-American prostate cancer survivors and their spouses. Psychooncology. 2011; doi: 106-10. doi: 10.1002/pon.1711.

10. Cayless S, Forbat L, Illingworth N, Hubbard G, \& Kearney N,. Men with prostate cancer over the first year of illness: their experiences as biographical disruption. Support Care Cancer. 2010; doi: 10.1007/s00520-009-0624-4.

11. Freedman ML, Haiman CA, Patterson N, McDonald GJ, et al. Admixture mapping identifies 8q24 as a prostate cancer risk locus in African-American men. Proc Natl Acad Sci USA. 2006; 10.1073/pnas.0605832103.

12. Jones RA, Taylor AG, Bourguignon C, Steeves R, et al. Family interactions among African American prostate cancer survivors. Fam Community Health. 2008; doi:

10.1097/01.FCH.0000324478.55706.fe.

13. Strauss AL. Qualitative analysis for social scientists. New York: Cambridge University Press; 1987.

14. Strauss A, Corbin J. Discovery of grounded theory. New York: Routledge; 2017.

15. Miles MB, Huberman AM. Qualitative data analysis: An expanded sourcebook. 2nd ed. Thousand Oaks, CA: SAGE Publications, Inc; 1994.

16. Charmaz K. Constructing grounded theory. Los Angeles: SAGE Publications, Inc; 2014.

17. Brown CM \& Segal R. Ethnic differences in temporal orientation and its implications for hypertension management. J Health Soc Behav. 1996;37(4):350-61.

18. Holman EA \& Silver RC. Getting" stuck" in the past: temporal orientation and coping with trauma. Journal of personality and social psycholog 1998;74(5):1146.

19. Odedina F, Young ME, Pereira D, Williams C, Nguyen J, \& Dagne G. Point of prostate cancer diagnosis experiences and needs of black men: the Florida CaPCaS study. J of Community and Supportive Oncology. 2017;15(1):10-19.

20. Odedina FT. Needs of Black Men at the Point of Prostate Cancer Di-agnosis (PPCD): The Florida CaPCaS Study. Int J Cancer Oncol. 2017; doi: 10:2377-0902.

21. Odedina FT, Yu D, Akinremi TO, Reams RR, Freedman ML, \& Kumar N. Prostate cancer cognitivebehavioral factors in a West African population. Journal of immigrant and minority health. 2009;11(4):258-267. 
22. Daniel MG. Knowledge and perceptions of prostate cancer among Nigerian male immigrants. ABNF Journal. 2013;24(1):23.

23. Woods VD, Montgomery SB, Belliard JC, Ramírez-Johnson J, \& Wilson CM. Culture, black men, and prostate cancer: What is reality? Cancer Control. 2004;11(6):388-396.

24. Blocker DE, Romocki LS, Thomas KB, et al. Knowledge, beliefs and barriers associated with prostate cancer prevention and screening behaviors among African-American men. Journal of the National Medical Association. 2006;98(8):1286.

25. Wray RJ, McClure S, Vijaykumar S, et al. Changing the conversation about prostate cancer among African Americans: results of formative research. Ethnicity \& health. 2009;14(1):27-43.

26. Allen JD, Mohllajee AP, Shelton RC, Drake BF, \& Mars DR. A computer-tailored intervention to promote informed decision making for prostate cancer screening among African American men. 2009; doi: $10.1177 / 1557988308325460$.

27. Lepore SJ, Wolf RL, Basch CE, et al. Informed decision making about prostate cancer testing in predominantly immigrant black men: a randomized controlled trial. Annals of Behavioral Medicine. 2012; doi: 10.1007/s12160-012-9392-3.

28. Webb CR, Kronheim L, Williams JE, \& Hartman TJ. An evaluation of the knowledge, attitudes, and beliefs of African-American men and their female significant others regarding prostate cancer screening. Ethnicity and Disease. 2006;16(1):234.

29. Pedersen VH, Armes J, \& Ream E. Perceptions of prostate cancer in Black African and Black Caribbean men: a systematic review of the literature. Psycho-oncology. 2012;21(5):457-468.

30. Holt CL, Wynn TA, Southward P, Litaker MS, Jeames S, \& Schulz E. Development of a spiritually based educational intervention to increase informed decision making for prostate cancer screening among church-attending African American men. Journal of health communication. 2009;14(6):590604.

31. Halbert $\mathrm{CH}$, Weathers $\mathrm{B}$, Delmoor $\mathrm{E}$, et al. Racial differences in medical mistrust among men diagnosed with prostate cancer. Cancer. 2009;115(11):2553-2561.

32. Odedina FT, Akinremi TO, Chinegwundoh C, et al. Does the prostate cancer disparities seen in US Black men follow the path of the Transatlantic Slave Trade?. Infect Agent Cancer. 2009;4(Suppl 1):S2.

33. Kumar NB, Yu D, Akinremi TO, \& Odedina FT. Comparing dietary and other lifestyle factors among immigrant Nigerian men living in the US and indigenous men from Nigeria: potential implications for prostate cancer risk reduction. Journal of immigrant and minority health. 2009;11(5):391-399.

34. Odedina FT, Yu D, Akinremi T, Reams RR, Freedman ML, \& Kumar N. Cultural Beliefs and Values among Black Men of West African Ancestry: Potential Impact on Prostate Cancer Prevention and Early Detection. 2009.

\section{Figures}




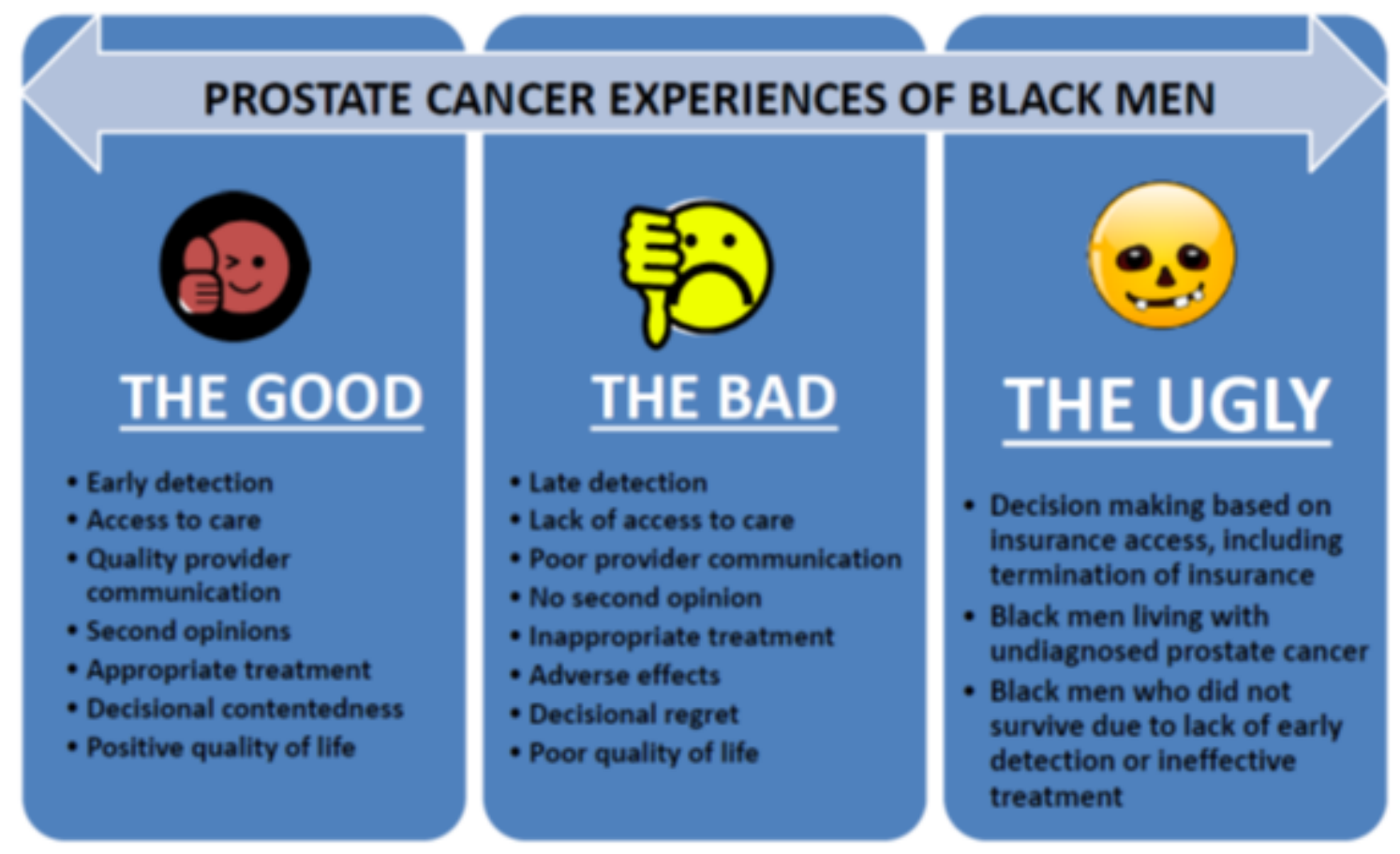

\section{Figure 1}

Prostate Cancer Experiences of Black Men: The Good, The Bad and The Ugly The experiences of the Black prostate cancer survivors across the prostate cancer care continuum were categorized into "the good", "the bad" and "the ugly".

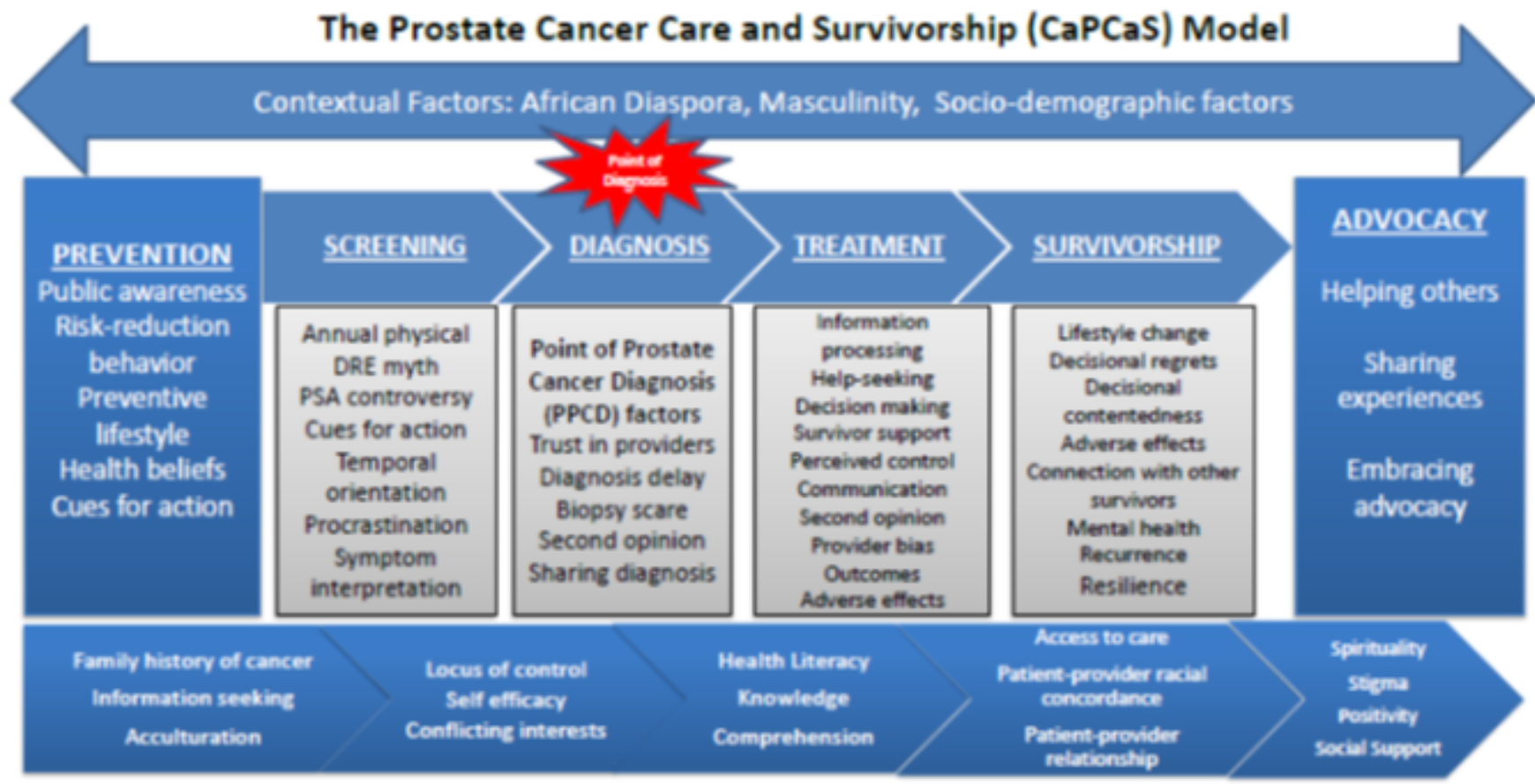

Cross-Cutting Factors

Figure 2 
The Prostate Cancer Care and Survivorship (CaPCaS) Model A Prostate Cancer Care and Survivorship (CaPCaS) model was created representing the trajectory of prostate cancer prevention, screening, diagnosis, treatment, survivorship, and advocacy.

\section{Supplementary Files}

This is a list of supplementary files associated with this preprint. Click to download.

- CaPCaSSupplementaryDataJCS.pdf 